\title{
Short-term Effects of Overnight Orthokeratology on Corneal Sub-basal Nerve Plexus Morphology and Corneal Sensitivity
}

\author{
María Nombela-Palomo, M.Sc., Gema Felipe-Marquez, M.Sc., Jose Luis Hernandez-Verdejo, Ph.D., \\ and Amelia Nieto-Bona, Ph.D.
}

\begin{abstract}
Objective: To assess the effects of a short period of orthokeratology (OK) on corneal subbasal nerve plexus (SBNP) morphology and corneal sensitivity. Methods: Measurements were made in 56 right eyes of 56 subjects with low-to-moderate myopia who wore 2 OK lens designs (Group CRT: HDS 100 Paragon CRT, $n=35$; Group SF: Seefree; $n=21$ ) for a period of 1 month and in 15 right eyes of noncontact lens wearers as controls. The variables determined in each participant were corneal sensitivity using a Cochet-Bonnet esthesiometer and 12 SBNP variables determined on laser scanning confocal microscopy images using 3 different software packages. Correlation between SBNP architecture and corneal sensitivity was also examined.

Results: Few changes were observed over the 1-month period in the variables examined in the OK treatment and control groups. However, significant reductions were detected over time in the number of nerves in the central cornea in the groups CRT $(P=0.029)$ and SF $(P=0.043)$ and in central corneal sensitivity in CRT $(P=0.047)$ along with significant increases in central and midperipheral corneal Langerhans cell counts in SF ( $P=0.001$ and 0.048 , respectively).

Conclusions: This study provides useful data to better understand the anatomical changes induced by $\mathrm{OK}$ in corneal SBNP. The different response observed to the $2 \mathrm{OK}$ lens designs requires further investigation.
\end{abstract}

Key Words: Subbasal nerve plexus-Corneal sensitivity-Overnight orthokeratology—Confocal microscopy—Esthesiometer.

(Eye \& Contact Lens 2016;0: 1-8)

$\mathrm{C}$ orneal refractive therapy (CRT), more commonly known as overnight orthokeratology (OK), is a treatment option for individuals with mild or moderate myopia that has so far provided predictable outcomes with accurate correction of refractive errors. ${ }^{1-3}$ This form of treatment has consistent effects provided that specially designed OK lenses are regularly used overnight ${ }^{4,5}$ to improve uncorrected visual acuity ${ }^{1-3}$ and thus offer clear unassisted vision throughout the day.

From the Department of Optics II (Optometry and Vision) (M.N.-P., G.F.-M.), Faculty of Optics and Optometry, Complutense University of Madrid, Spain; Department of Optics II (Optometry and Vision) (J.L.H.-V., A.N.-B.), Faculty of Optics and Optometry, Contactology and Optometry Research Group (GICO), Complutense University of Madrid, Spain.

The authors have no funding or conflict of interests to disclose.

Address correspondence to María Nombela-Palomo, M.Sc., Department of Optics II (Optometry and Vision), Faculty of Optics and Optometry, Complutense University of Madrid, Arcos de Jalón 118, Madrid 28037, Spain; e-mail: mnombela@ucm.es

Accepted April 1, 2016.

DOI: 10.1097/ICL.0000000000000282
Over the last 2 decades, significant progress has been made in understanding the changes that take place in response to $\mathrm{OK}$. However, although its effects on corneal topography, visual acuity, contrast sensitivity, optical quality of the eye, and biomechanical properties have been widely investigated, ${ }^{5-10}$ only a few studies have examined the histological effects of this technique on the morphology of the human cornea. ${ }^{1-13}$ In 2011, Nieto-Bona et al. ${ }^{13}$ published the results of a year-long clinical study including individuals who wore OK lenses daily. This study reported corneal structural and optical changes. The irreversible thinning of the Bowman layer and subbasal nerve plexus (SBNP) observed by these authors prompted a further study ${ }^{14}$ which indicated that wearing OK lenses modifies the normal distribution of subbasal nerves. In addition, it has been confirmed that the tissue redistribution observed during OK treatment leads to increased levels of proinflammatory molecules. ${ }^{15}$ Moreover, inflammation in response to the use of $\mathrm{OK}$ lenses also seems to induce the migration of Langerhans cells to the cornea. ${ }^{16,17}$ Bearing these 2 factors in mind, it is reasonable to predict Langerhans cell density changes in the cornea with the use of OK lenses along with a deficit in corneal nerve function $^{18}$ which could be detected by measuring corneal sensitivity.

To date, the relationship between corneal architecture and sensitivity has been examined in contact lens wearers, ${ }^{19}$ patients who have undergone corneal surgery ${ }^{20}$ and patients with systemic disease. $^{21,22}$ In a study conducted in a large population of noncontact lens wearers, Patel et al. ${ }^{23}$ observed correlation between SBNP density or SBNP tortuosity and corneal sensitivity. More recently, Lum et al. ${ }^{24}$ reported nerve fiber redistribution and corneal sensitivity changes in OK patients based on SBNP appearance in $2 \mathrm{OK}$ subjects and suggested that OK leads to modified SBNP distribution in the central and midperipheral cornea.

Given the important role of corneal nerves in corneal sensation, wound healing, epithelial cell proliferation, epithelial integrity and protection along with the immune role of Langerhans cells, indepth knowledge is required of any changes to these structures induced by $\mathrm{OK}$ and possible effects of such changes on corneal sensitivity. The morphology of the SBNP and Langerhans cells can be easily determined by in vivo laser scanning confocal microscopy (IVCM). This noninvasive technique has shown good repeatability. ${ }^{25}$

This study was designed to prospectively and objectively assess the short-term response to OK by determining effects on corneal nerve morphology as determined by IVCM and possible correlations with corneal sensitivity measured using a Cochet-Bonnet esthesiometer. A further aim was to confirm that different OK lens 
designs had similar effects on the human cornea. Our working hypothesis was that OK lenses affect corneal morphology including effects on the SBNP layer and on central and midperipheral corneal sensitivity.

\section{METHODS}

The study protocol (prospective, longitudinal, single-center) adhered to the tenets of the Declaration of Helsinki and received approval from the Ethics Committee of the Hospital Carlos III (Madrid, Spain). Written informed consent was obtained from each participant.

\section{Sample Size Calculation}

Based on the SBNP density results obtained by Patel et al., ${ }^{23}$ we used Granmo 7.12 software to calculate the sample size required for this study. For an alpha risk of 0.05 and beta risk of 0.2 in a 2tailed test, it was calculated that 20 subjects would be needed in the treatment group and 10 in the control group to detect a significant difference equal or greater than $3,366 \mu \mathrm{m} / \mathrm{mm}^{2}$ and a correlation coefficient between the initial and final measurement of 0.8 . The dropout rate anticipated was of $15 \%$.

\section{Subjects}

Participants were enrolled if they had no systemic or eye disease, history of eye surgery, and evidence of keratoconus or corneal irregularity. Pregnant women and those planning to become pregnant throughout the study were excluded along with rigid gas-permeable contact lens users. Hydrophilic lens users were instructed to remove their contact lenses 4 weeks before starting the study.

Of 161 individuals initially contacted who signed the informed consent form and were subjected to preliminary tests, 36 withdrew for personal reasons and $25 \mathrm{did}$ not meet the inclusion criteria.

The 100 remaining subjects were randomly assigned to wear the Paragon CRT lenses (composition HDS 100, diameter $10.5 \mathrm{~mm}$; Paragon Vision Sciences, Interlenco, Madrid, Spain) $(C R T, n=40)$ or Seefree lenses (composition Boston XO2, diameter $10.8 \mathrm{~mm}$; Conóptica, Barcelona, Spain) (SF, $\mathrm{n}=40$ ) in both eyes. The control group was comprised of subjects $(n=20)$ who were not contact lens wearers.

Of these 100 subjects, 29 withdrew from the study: 3 in CRT and 5 in SF because of poor uncorrected visual acuity improvement, and 2 in CRT and 4 in SF because of a grade 2 staining pattern (Efron grade scale). In addition, $10 \mathrm{SF}$ group and 5 control subjects could not attend the follow-up visit.

The final study population was made up of 71 subjects aged 18 to 30 years (CRT, 35 subjects aged $23.9 \pm 3.7$ years; SF, 21 subjects aged 25.8 \pm 4.1 years; and Control, 15 subjects aged 25.6 \pm 3.7 years). Subjective refraction was sphere -0.50 to -5.00 diopters (D) and cylinder -0.25 to $-1.25 \mathrm{D}$.

Patients were examined in 2 separate visits at baseline and 1 month after starting OK treatment. In each visit, 2 tests were performed by a single experienced clinician between 2 and $4 \mathrm{hr}$ after removing the lenses: corneal sensitivity and corneal morphology using the corneal module Rostock Corneal Module (RCM) of the Heidelberg Retina Tomograph II (HRT; Heidelberg Engineering $\mathrm{GmbH}$, Heidelberg, Germany).

For the first test, we used a Cochet-Bonnet esthesiometer (Luneau, Paris, France) mounted on a slitlamp microscope to obtain sensitivity measurements at the corneal apex and $3 \mathrm{~mm}$ temporal from the apex. To take the central measurements, participants were asked to stare at a point in the slitlamp microscope aligned with their visual axis. For the midperipheral measurements, subjects stared at a point $30 \mathrm{~cm}$ away from their eyes. Eye rotation was $0.6^{\circ}$ nasal. The Cochet-Bonnet esthesiometer consists of a $0.12 \mathrm{~mm}$ diameter nylon monofilament of variable length $(0-60 \mathrm{~mm})$ that applies pressure on the cornea in the range 11 to $200 \mathrm{mg} / 0.0113 \mathrm{~mm}^{2}$. The criterion of first visible bending was used to standardize the applied pressure. To refine the accuracy of the measurements taken, the esthesiometer was mounted on a slitlamp microscope as described previously. ${ }^{26}$

For the IVCM procedure, 2 videos were obtained in each patient, the first of the central cornea and the second of the midperipheral cornea. Each video included a sequence of images spanning from the superficial epithelium to the anterior stroma. Besides these test sessions, standard visits for OK lens fitting were also scheduled. The clinical procedures and OK lens adaptation followed have been formerly described ${ }^{3}$ and included fluorescein staining at each visit to assess ocular surface health.

\section{SBNP Image Acquisition}

In vivo laser scanning confocal microscopy was conducted on the right eye of each participant using the RCM corneal module. The instrument was set up using standard techniques. ${ }^{27-29}$ During the examination, the subject was asked to stare at 2 different points on 2 different occasions: first, the central cornea was assessed while they stared at a central point set inside the instrument. To obtain the midperipheral measurements, patients were asked to look at a point $1 \mathrm{~m}$ away from their eyes. In this second setup, eye rotation was $6.20^{\circ}$ superonasal and thus the center of the corneal section obtained was $2.4 \mathrm{~m}$ below corneal apex and $3.2 \mathrm{~mm}$ temporal to the corneal apex. During the baseline examination, all subjects wore a disposable contact lens (Proclear 1 Day CooperVision, Madrid, Spain) in the contralateral (left) eye to allow them to focus on the fixation spot with the eye under test. In the second test session, all patients could clearly see the fixation spot because of the OK treatment.

The RCM was set on "volume mode," also called z-scan, to record a video of the different layers of the cornea down to a depth of $80 \mu \mathrm{m}$. For the test to be valid, the fixation spot must not change during the examination. The Z-scan mode allows the operator to identify fixation changes, increasing the reliability of measurements. Each test took only $6 \mathrm{~s}$ thus minimizing the number of discarded videos because of fixation changes. The videos were transformed into series of 40 bi-dimensional images $(250 \times 250$ $\mu \mathrm{m})$. The groups of images were then randomly assessed in a masked fashion.

\section{Image Analysis}

Image analysis was conducted by a single experienced examiner using 3 different software packages: ImageJ (www.rsb.info.nih. gov/ij), NeuronJ (imagescience.org/meijering/software/neuronj/) and Matlab (MatLab; The Mathworks, Natick, MA). The 3 programs were used to objectively assess all possible SBNP parameters: ImageJ was used for SBNP thickness, total and local reflectivity, nerve density, width, orientation and length, and number of Langerhans cells; NeuronJ for nerve reflectivity and number of nerves; and MatLab for nerve tortuosity. 
From the 40 images extracted from each video, only those corresponding to the SBNP layer were processed. The thickness of the SBNP was defined as the distance between the first focused image obtained of the SBNP and the first focused image of basal cells as determined previously. ${ }^{13}$ Total SBNP reflectivity was measured by analyzing the histogram of each image using ImageJ. ${ }^{30}$ Subsequently, the best-focused image (the one with the best contrast) in each group was selected and, using the same histogram procedure as before, local reflectivity was obtained. Also using ImageJ, the following measurements were made on the bestfocused image representing an area of $62,500 \mu^{2}$ : nerve width, density, orientation and length, and density of Langerhans cells. Nerve width measurements were made at 4 equidistant points along the nerve length, and the orientation and length of every nerve in the frame was measured with the caliper tool in ImageJ iJ145 (Fig. 1A). Average values for each variable were recorded. The observation protocol was as described previously. ${ }^{13}$ In every picture, the lengths of all nerves in pixels per frame were converted into micrometers, added together and a density index defined as total nerve length per $\mathrm{mm}^{2}$. Langerhans cells were counted manually in each image by a single experienced observer according to the method used by Nieto-Bona et al. ${ }^{13}$ for epithelial cell counts (Fig. 1B).

Neuron $\mathrm{J}^{31}$ is a specific plug-in for ImageJ that allows for nerve tracing and measurement of nerve reflectivity (Fig. 2). Its reliability has been recently confirmed. ${ }^{32}$ Using this tool, we determined nerve reflectivity and number in the best-focused SBNP image. NeuronJ also provides nerve length measurements such that these could be compared with the ImageJ data. Nerve branches were not included in the counts. The coordinates of the nerves in the images were recorded to be processed at a later stage using MatLab for tortuosity measurements as described previously. ${ }^{33}$ In parallel, sub-

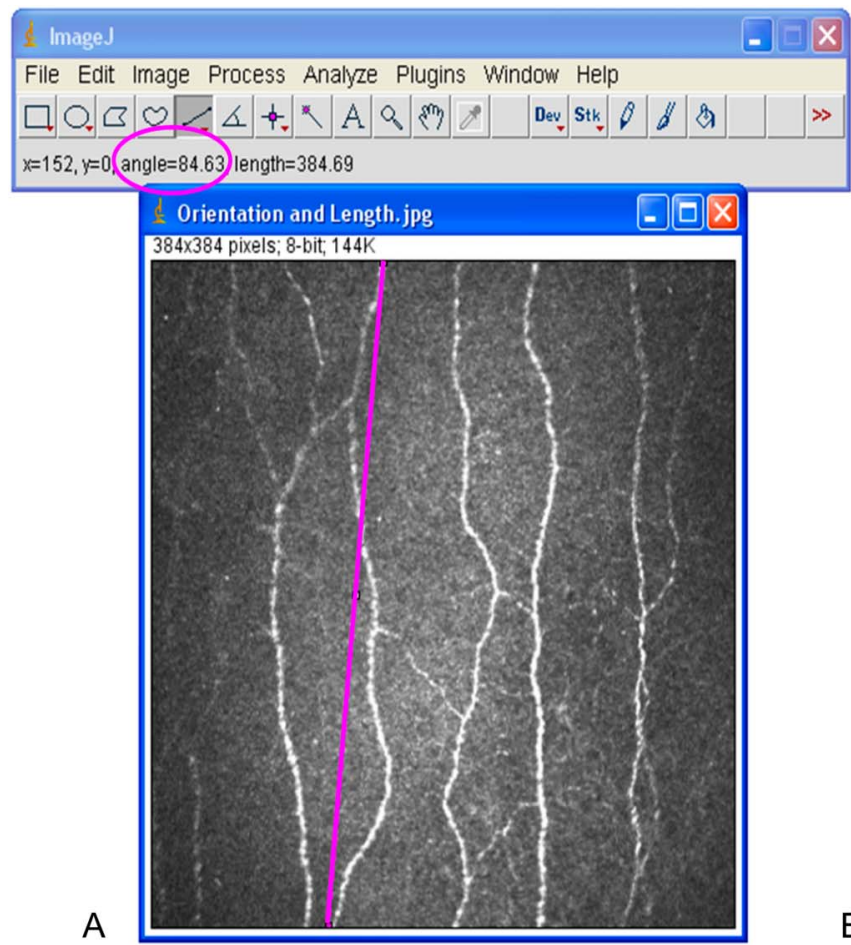

FIG. 1. (A) Subbasal nerve orientation and length jective tortuosity measurements were obtained following the procedure described by Oliveira-Soto and Efron. ${ }^{34}$

\section{Statistical Analysis}

Data were recorded in Excel (2003, Microsoft) and analyzed using the Statgraphics Centurion XVI package, version 16.1.17. Statistical analysis was performed on the data obtained for the right eye of each subject. All variables showed a Gaussian distribution according to the Kolmogorov-Smirnov test. Baseline and follow-up data were compared using a Student paired $t$ test and comparisons among groups were performed by one-way analysis of variance (ANOVA). Post hoc comparisons were made of mean values for the cornea center and periphery. All data are provided as the mean \pm SD. Significance was set at $P<0.05$.

\section{RESULTS}

The final study sample consisted of 71 healthy right eyes of 33 men and 38 women of mean age $( \pm$ SD) $24.8 \pm 3.9$ years. None of the OK lens wearers who completed the 1-month treatment period experienced any adverse events and no abnormalities of the eyes were detected by slitlamp microscopy. The baseline characteristics of the participants and mean refractive and corneal curvature data for the right eyes are provided in Table 1 by subject group.

The SBNP variables recorded in the 3 subject groups at baseline and after 1 month of $\mathrm{OK}$ for the central and midperipheral cornea are provided in Tables 2 and 3.

In each of the subject groups (CRT, SF and control), practically no differences were detected in any of the variables over time. The only significant differences observed from baseline to 1-month posttreatment were: 1) a reduction in the number of

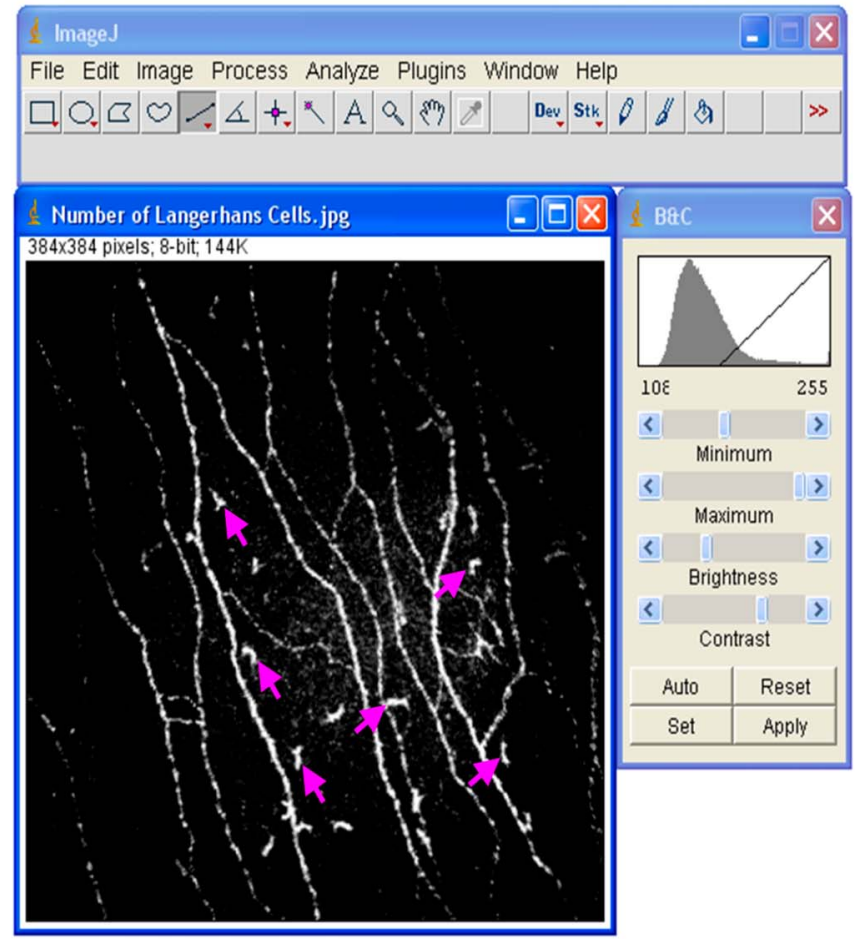

(B) Langerhans cells measured using ImageJ. 


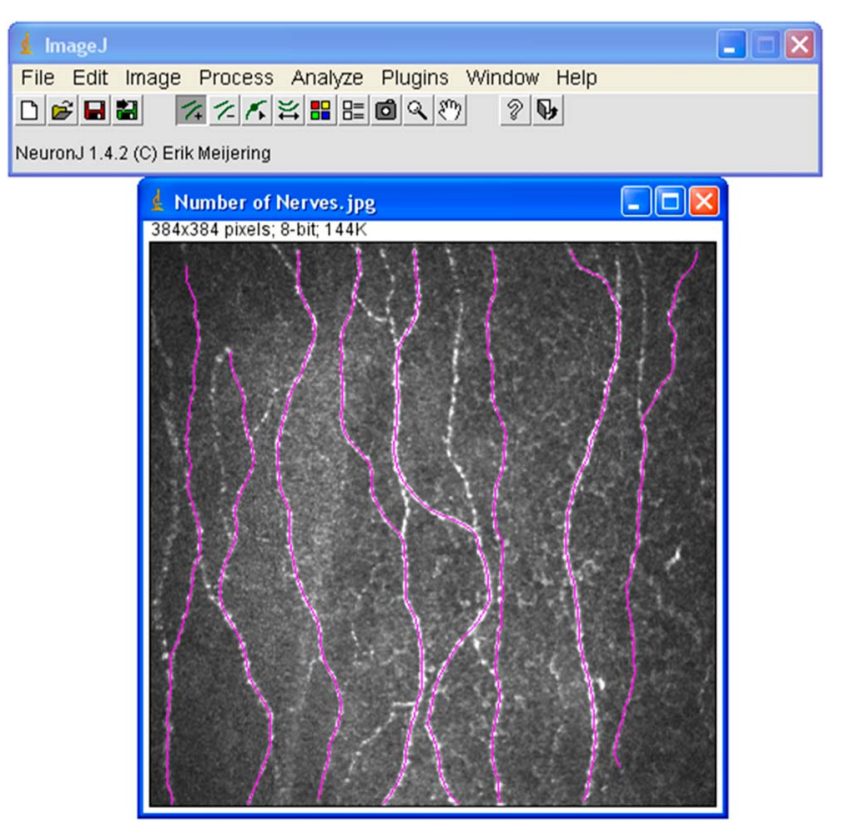

FIG. 2. Nerve tracing using NeuronJ. Number of nerves, average nerve length, and average nerve reflectivity (indicated by a circle in the image) are automatically displayed in a window.

nerves in the central cornea in the CRT group $(P=0.029)$ and SF group $(P=0.043) ; 2)$ a reduction in central corneal sensitivity in the CRT group $(P=0.047)$; and 3$)$ increases in Langerhans cell counts in the central and midperipheral cornea in SF $(P=0.001$ and 0.048 , respectively). These 3 main findings are illustrated in a bar chart (Fig. 3).

When these differences between baseline and 1 month values were analyzed by ANOVA, significant differences among the 3 groups emerged for the number of Langerhans cells $(P=0.041)$. No correlation was detected between the change produced in the Langerhans cell count or number of nerves and corneal sensitivity, both central and midperipheral.

Midperipheral corneal data could not be obtained in the control group. In many of these subjects, images were insufficiently clear for reliable SBNP measurements on the best-focused image. Thus, only sensitivity and SBNP thickness data were available for the midperipheral cornea in control subjects: corneal sensitivity was $4.94 \pm 0.42 \mathrm{~cm}$ at baseline and its change over the 1-month period was $-0.06 \pm 0.18 \mathrm{~cm}$; SBNP thickness was $12.11 \pm 1.27 \mu \mathrm{m}$ and its

TABLE 1. Refractive and Corneal Variables Previous to the OK Treatment

\begin{tabular}{lccc}
\hline Parameter & $\begin{array}{c}\text { Group CRT } \\
(\mathrm{n}=35), \mathrm{m} \pm \mathrm{SD}\end{array}$ & $\begin{array}{c}\text { Group Seefree } \\
(\mathrm{n}=21), \mathrm{m} \pm \mathrm{SD}\end{array}$ & $\begin{array}{c}\text { Group Control } \\
(\mathrm{n}=15), \mathrm{m} \pm \mathrm{SD}\end{array}$ \\
\hline Male/female ( $\mathrm{n})$ & $16 / 19$ & $8 / 13$ & $9 / 6$ \\
Age (yr) & $23.9 \pm 3.7$ & $25.81 \pm 4.14$ & $25.6 \pm 3.3$ \\
$\varnothing$ corneal & $12.4 \pm 0.3$ & $12.3 \pm 0.3$ & $12.4 \pm 0.2$ \\
Sphere (D) & $-2.10 \pm 0.96$ & $-2.11 \pm 0.96$ & $-2.89 \pm 1.30$ \\
Cylinder (D) & $-0.16 \pm 0.26$ & $-0.19 \pm 0.28$ & $-0.31 \pm 0.41$ \\
Sim K Klat (D) & $43.27 \pm 1.48$ & $43.36 \pm 1.32$ & $43.03 \pm 1.95$ \\
Sim K steep (D) & $44.02 \pm 1.56$ & $44.01 \pm 1.43$ & $43.63 \pm 1.76$ \\
\hline
\end{tabular}

$n$, number of subjects; $m$, mean; SD, standard deviation; $\varnothing$, diameter; Sim $\mathrm{K}_{\text {flat }}$ and $\mathrm{Sim} \mathrm{K}_{\text {steep, }}$ simulated keratometry readings along flatter and steeper meridians. change over the 1-month period was $0.33 \pm 1.50 \mu \mathrm{m}(P=0.35$ and $P=0.52$, respectively; Students paired $t$ test). Post hoc comparisons of means indicated no differences over time for central corneal sensitivity $(P=0.11)$, central length $(P=0.17)$, nerve density $(P=0.72)$, central and peripheral Langerhans cell density ( $P=0.53$ and $P=0.68$, respectively), and peripheral SBNP thickness $(P=0.46)$.

\section{DISCUSSION}

This study was designed to examine the SBNP in detail and identify possible changes produced in several SBNP variables in response to 1 month of OK lens wear. Our data indicate high agreement between the SBNP thickness and remaining variables recorded here and reported ranges for the normal cornea. ${ }^{13,19,34}$

Our treatment (CRT and SF) and control groups varied in terms of the numbers and distributions of Langerhans cells observed both at baseline and at the end of the 1-month study period. In individuals wearing Seefree OK lenses, Langerhans cells were mainly observed in the midperipheral cornea and more rarely seen in central cornea, in agreement with the findings of others. ${ }^{17,35}$ In both these areas, Langerhans cell density was greater after 1 month of treatment in this group. The immunological functions of dendritic cells and their role in corneal inflammation and dry eye disease have been well established. ${ }^{36,37}$ Thus, it is reasonable to assume that the cornea will respond to a contact lens as it would to a foreign body by increasing Langerhans cell numbers. This response is likely triggered by wear-associated mechanical irritation, hypoxia, and mediator release. It would be interesting to confirm whether Langerhans cell counts fall after OK lens removal. Our study participants were instructed to remove their contact lenses (if worn) 4 weeks before the start of OK treatment to ensure that Langerhans cell levels matched normal levels for the bare cornea. However, baseline Langerhans cell counts were slightly higher in both our treatment groups than those reported for healthy individuals by Zhivov et al. ${ }^{17}$ and Sindt et al. ${ }^{38}$ We speculate that this washout period of 4 weeks was insufficient or perhaps participants failed to follow the instructions given by the research group. In contrast, no significant differences in Langerhans cell numbers were recorded after wearing the CRT lenses. These lenses vary in their geometry. As an explanation for the lack of change observed in Langerhans cells after treatment in the CRT group, we propose that the larger diameter of this lens (10.8 vs. $10.5 \mathrm{~mm})$ may have determined that counts were made outside the return zone. This hypothesis is supported by the change in mean corneal radii observed in the tangential topography map after treatment in both the CRT and SF groups (Fig. 4), which is directly linked to the pressure the lens generates against the cornea. As may be observed, the behavior of the inferior zone where the confocal microscopy measurements were taken was slightly different, though not significantly $(P>0.05)$, between the 2 lens types. Hence, the pressure exerted by the CRT lens was more positive than that observed for the SF lens and could, at least in part, explain the differences in Langerhans cells noted. According to Zhivov, in healthy eyes these cells appear at greater densities in the periphery than the central corneal zone. From the report by this author, a positive centralperipheral gradient of $37 \%$ may be deduced. Our results indicate similar gradients for both lenses (78\% for CRT and $81 \%$ for SF), which were nevertheless higher than the values cited by Zhivov, 
TABLE 2. Parameter Values Previous to OK Treatment and Changes Produced in Central Cornea After 1 Month of Continuous OK Treatment

\begin{tabular}{|c|c|c|c|c|c|c|}
\hline \multirow[b]{2}{*}{ Central Cornea } & \multicolumn{3}{|c|}{ CRT $(n=35)$} & \multicolumn{3}{|c|}{ Seefree $(n=21)$} \\
\hline & Baseline $(m \pm S D)$ & $\Delta(\mathrm{m} \pm \mathrm{SD})$ & $P$ & Baseline $(m \pm S D)$ & $\Delta(\mathrm{m} \pm \mathrm{SD})$ & $P$ \\
\hline Corneal sensitivity $(\mathrm{cm})$ & $5.04 \pm 0.72$ & $-0.23 \pm 0.66$ & $<0.05^{a}$ & $5.45 \pm 0.50$ & $-0.05 \pm 0.67$ & 0.75 \\
\hline SBNP thickness $(\mu \mathrm{m})$ & $10.27 \pm 2.01$ & $0.24 \pm 3.59$ & 0.76 & $10.67 \pm 2.09$ & $-0.23 \pm 2.86$ & 0.78 \\
\hline Reflectivity $_{\text {Global }}$ & $0.33 \pm 0.06$ & $-0.01 \pm 0.12$ & 0.50 & $0.32 \pm 0.10$ & $-0.01 \pm 0.10$ & 0.42 \\
\hline Reflectivity Local & $0.36 \pm 0.10$ & $-0.02 \pm 0.15$ & 0.20 & $0.35 \pm 0.15$ & $-0.01 \pm 0.15$ & 0.51 \\
\hline Nerve reflectivity & $0.76 \pm 0.03$ & $-0.02 \pm 0.04$ & 0.47 & $0.76 \pm 0.03$ & $-0.03 \pm 0.04$ & 0.43 \\
\hline Length $(\mu \mathrm{m})$ & $231.87 \pm 40.16$ & $-5.15 \pm 66.63$ & 0.59 & $194.29 \pm 66.63$ & $5.88 \pm 80.32$ & 0.63 \\
\hline Nerve density $\left(\mu \mathrm{m} / \mathrm{mm}^{2}\right)$ & $11,152.60 \pm 5,011.05$ & $-1,956.38 \pm 5,307.39$ & 0.07 & $9,680.69 \pm 4,214.51$ & $-1,281.74 \pm 7,304.94$ & 0.45 \\
\hline Nerves (n) & $4.59 \pm 1.87$ & $-0.92 \pm 2.04$ & $<0.05^{a}$ & $4.81 \pm 1.63$ & $-1.26 \pm 2.54$ & $<0.05^{a}$ \\
\hline Orientation (degrees) & $93.67 \pm 28.51$ & $3.74 \pm 30.51$ & 0.54 & $94.65 \pm 27.35$ & $8.62 \pm 38.69$ & 0.34 \\
\hline Langerhans density $\left(\mu \mathrm{m} / \mathrm{mm}^{2}\right)$ & $70.40 \pm 46.88$ & $-1.28 \pm 36.16$ & 0.86 & $54.08 \pm 29.28$ & $21.60 \pm 24.00$ & $<0.05^{a}$ \\
\hline Width $(\mu \mathrm{m})$ & $2.43 \pm 0.40$ & $-0.08 \pm 0.44$ & 0.37 & $2.31 \pm 0.44$ & $-0.10 \pm 0.26$ & 0.10 \\
\hline Tortuosity objetive & $22.32 \pm 9.95$ & $-4.64 \pm 13.41$ & 0.09 & $21.94 \pm 11.63$ & $-4.53 \pm 16.89$ & 0.26 \\
\hline \multirow[t]{2}{*}{ Tortuosity $_{\text {subjetive }}$} & $1.55 \pm 0.89$ & $0.19 \pm 1.13$ & 0.39 & $1.52 \pm 0.87$ & $-0.05 \pm 0.78$ & 0.77 \\
\hline & & \multicolumn{5}{|c|}{ Control $(n=15)$} \\
\hline Central Cornea & & Baseline $(\mathrm{m} \pm \mathrm{SD})$ & & $\Delta(\mathrm{m} \pm \mathrm{SL}$ & & $P$ \\
\hline Corneal sensitivity $(\mathrm{cm})$ & & $5.06 \pm 0.35$ & & $0.06 \pm 0.2$ & & 0.13 \\
\hline SBNP thickness $(\mu \mathrm{m})$ & & $9.88 \pm 1.27$ & & $0.44 \pm 1.1$ & & 0.27 \\
\hline Reflectivity & & $0.28 \pm 0.18$ & & $0.01 \pm 0.0$ & & 0.47 \\
\hline Reflectivity Local & & $0.30 \pm 0.20$ & & $0.01 \pm 0.0$ & & 0.71 \\
\hline Nerve reflectivity & & $0.29 \pm 0.02$ & & $0.04 \pm-0$ & & 0.15 \\
\hline Length $(\mu \mathrm{m})$ & & $240.19 \pm 50.78$ & & $28.91 \pm 50$ & & 0.12 \\
\hline Nerve density $\left(\mu \mathrm{m} / \mathrm{mm}^{2}\right)$ & & $11,227.10 \pm 5,912.91$ & & $4,214.82 \pm 8,0$ & .28 & 0.26 \\
\hline Nerves (n) & & $4.50 \pm 2.10$ & & $1.00 \pm 1.8$ & & 0.30 \\
\hline Orientation (degrees) & & $89.20 \pm 37.39$ & & $4.20 \pm 39$ & & 0.82 \\
\hline Langerhans density $\left(\mu \mathrm{m} / \mathrm{mm}^{2}\right)$ & & $37.76 \pm 37.53$ & & $3.20 \pm 7.6$ & & 0.37 \\
\hline Width $(\mu \mathrm{m})$ & & $2.44 \pm 0.91$ & & $0.20 \pm 0.9$ & & 0.64 \\
\hline Tortuosity objetive & & $21.34 \pm 11.95$ & & $11.73 \pm 9.7$ & & 0.06 \\
\hline Tortuosity $_{\text {subjetive }}$ & & $2.40 \pm 0.55$ & & $0.40 \pm 0.5$ & & 0.18 \\
\hline
\end{tabular}

Positive values shown in the $\Delta$ column correspond to a rise in the parameter value.

${ }^{a}$ Statistically significant differences between baseline and 1-month measurements for each pair of data.

$\mathrm{n}$, number of subjects/items; $\mathrm{m}$, mean; SD, standard deviation; $\Delta$, difference between baseline and 1 month after continuous OK treatment; $\mathrm{cm}$, centimeters; $\mu \mathrm{m}$, microns; $\mathrm{mm}^{2}$, squared millimeters.

suggesting a not so peripheral measurement point used by this author. However, although the positive gradient observed for the SF lenses was expected, ${ }^{17}$ that is, more cells in the central cornea than periphery, the opposite was observed for the CRT lenses. The different response to the 2 lens designs could be attributable to a measurement artifact given the small corneal zone worked with in the IVCM procedure.

Another interesting finding was a significant reduction in the number of nerves observed in the central cornea in both groups after 1 month of OK treatment. Nieto-Bona et al. ${ }^{11,13}$ also noted

TABLE 3. Parameter Values Previous to the OK treatment and Changes Produced in Mid-Peripheral Cornea After 1 Month of Continuous OK Treatment

\begin{tabular}{|c|c|c|c|c|c|c|}
\hline \multirow[b]{2}{*}{ Mid-Peripheral Cornea } & \multicolumn{3}{|c|}{ CRT $(n=35)$} & \multicolumn{3}{|c|}{ Seefree $(n=21)$} \\
\hline & Baseline $(m \pm S D)$ & $\Delta(\mathrm{m} \pm \mathrm{SD})$ & $P$ & Baseline $(\mathrm{m} \pm \mathrm{SD})$ & $\Delta(\mathrm{m} \pm \mathrm{SD})$ & $P$ \\
\hline Corneal sensitivity $(\mathrm{cm})$ & $5.29 \pm 0.67$ & $-0.01 \pm 0.08$ & 0.32 & $5.41 \pm 0.51$ & $-0.07 \pm 0.33$ & 0.33 \\
\hline SBNP thickness $(\mu \mathrm{m})$ & $12.09 \pm 4.41$ & $-2.47 \pm 5.28$ & 0.09 & $10.75 \pm 1.65$ & $0.46 \pm 2.18$ & 0.40 \\
\hline Reflectivity $_{\text {Global }}$ & $0.33 \pm 0.06$ & $-0.01 \pm 0.09$ & 0.13 & $0.33 \pm 0.08$ & $0.01 \pm 0.08$ & 0.59 \\
\hline Reflectivity & $0.35 \pm 0.08$ & $-0.01 \pm 0.12$ & 0.20 & $0.36 \pm 0.12$ & $-0.00 \pm 0.10$ & 0.96 \\
\hline Nerve reflectivity & $0.77 \pm 0.03$ & $-0.03 \pm 0.04$ & 0.34 & $0.77 \pm 0.03$ & $0.01 \pm 0.04$ & 0.72 \\
\hline Length $(\mu \mathrm{m})$ & $220.23 \pm 66.34$ & $-15.36 \pm 83.50$ & 0.37 & $217.71 \pm 42.99$ & $14.20 \pm 68.58$ & 0.41 \\
\hline Nerve density $\left(\mu \mathrm{m} / \mathrm{mm}^{2}\right)$ & $10,668 \cdot 10 \pm 5,369.93$ & $-2,141.66 \pm 7,087.29$ & 0.14 & $10,818.50 \pm 5,154.45$ & $458.36 \pm 6,280.06$ & 0.77 \\
\hline Nerves $(n)$ & $4.56 \pm 1.58$ & $-0.76 \pm 2.05$ & 0.08 & $4.55 \pm 1.76$ & $0.18 \pm 2.13$ & 0.74 \\
\hline Orientation (degrees) & $96.14 \pm 27.51$ & $7.29 \pm 35.90$ & 0.32 & $99.61 \pm 28.71$ & $7.47 \pm 52.48$ & 0.57 \\
\hline Langerhans density $\left(\mu \mathrm{m} / \mathrm{mm}^{2}\right)$ & $55.36 \pm 35.68$ & $-1.28 \pm 42.40$ & 0.88 & $66.40 \pm 33.28$ & $14.24 \pm 28.48$ & $<0.05^{a}$ \\
\hline Width $(\mu \mathrm{m})$ & $2.43 \pm 0.31$ & $-0.14 \pm 0.35$ & 0.06 & $2.25 \pm 0.24$ & $-0.01 \pm 0.28$ & 0.98 \\
\hline Tortuosity objetive & $20.75 \pm 10.06$ & $-2.19 \pm 12.22$ & 0.47 & $21.94 \pm 11.62$ & $-1.01 \pm 15.72$ & 0.78 \\
\hline Tortuosity $_{\text {subjetive }}$ & $1.52 \pm 0.65$ & $-0.04 \pm 0.73$ & 0.79 & $1.65 \pm 0.81$ & $0.12 \pm 0.69$ & 0.50 \\
\hline
\end{tabular}

Positive values shown in the $\Delta$ column correspond to a rise in the parameter value.

${ }^{a}$ Statistically significant differences between baseline and 1-month measurements for each pair of data.

$\mathrm{n}$, number of subjects/items; m, mean; SD, standard deviation; $\Delta$, difference between baseline and 1 month after continuous OK treatment; $\mathrm{cm}$, centimeters; $\mu \mathrm{m}$, microns; $\mathrm{mm}^{2}$, squared milimmeters. 
FIG. 3. Graphs a to $c$ represent the mean values ( \pm $\mathrm{SD}$ ) of the nerve parameters (shown on $y$-axis) quantified objectively in this study for the 3 subject groups in central $\left(a_{1}-c_{1}\right)$ and peripheral $\left(a_{\| 1}-c_{\| 1}\right)$ corneal areas. The height of the bars represents average values and the error bars represent standard deviations. Statistically significant differences are indicated by $\left(^{*}\right)$.
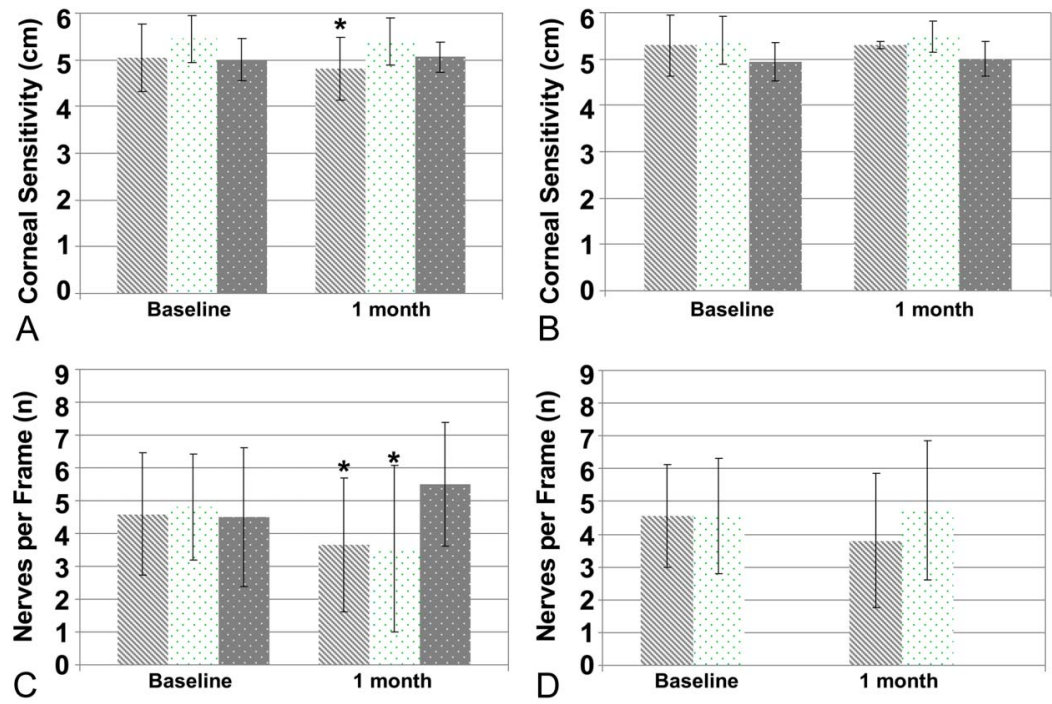

B
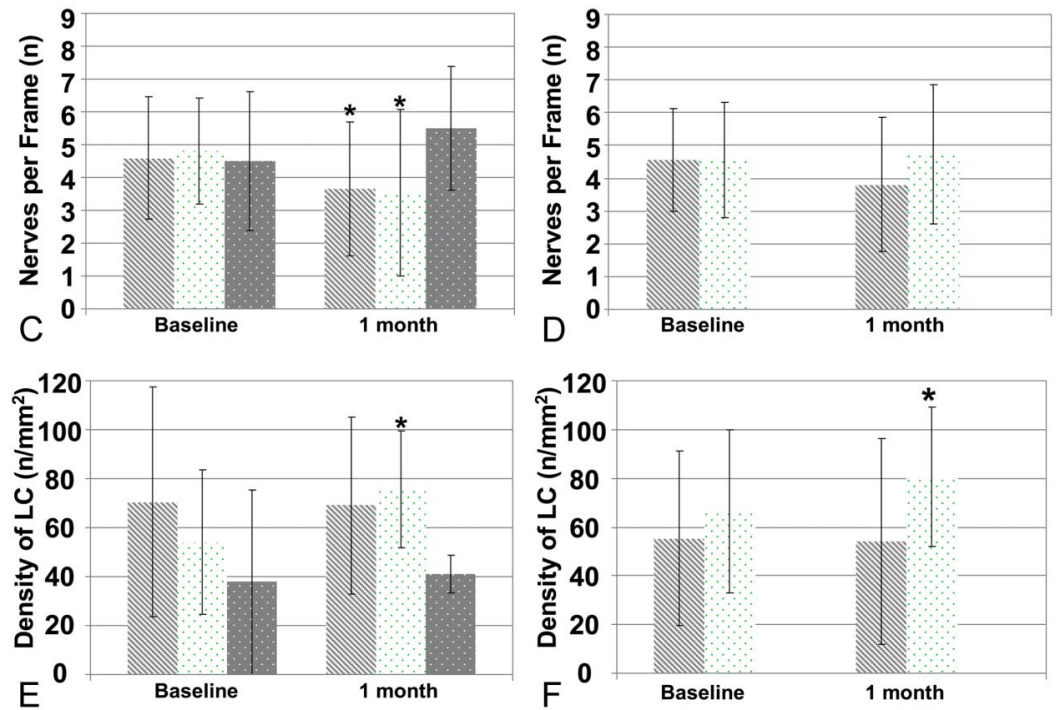

a downward trend in this factor using the same procedure to assess nerve density although we used an objective tool (NeuronJ) for this purpose. This finding is consistent with observations by Lum et al. ${ }^{14}$ who described the redistribution of subbasal nerve fibers toward the periphery leaving a sparsely populated central cornea. In line with this finding, the number of fibers in the midperipheral cornea showed an upward trend yet this increase was not significant possibly because our midperipheral measurements were made close to the return zone.

Correlation between nerve fibers and Langerhans cells is a topic of ongoing discussion. Auran et al. ${ }^{39}$ provided evidence of axon growth in the SBNP for the first time, raising the possibility that both nerve fibers and Langerhans cells migrate centripetally in tandem. Subsequent to this, Torii et al. ${ }^{40}$ reported correlation between Langerhans cells and epidermal nerves, whereas $\mathrm{He}$ et al. ${ }^{35}$ observed scarce contact between dendritic cells and corneal nerves in ex vivo corneas. Although no such correlation was detected here, the changes produced both in Langerhans cell and nerve numbers in the midperipheral cornea are compatible with the notion that Langerhans cells use nerve structures to move inside the cornea. ${ }^{39}$

The corneal sensitivity values recorded here were within normal limits, though slightly lower than reported for soft contact lens wearers ${ }^{19}$ and normal corneas. ${ }^{41}$ No significant intergroup differences in this variable were observed at baseline. However, a significant, but modest, decrease in central corneal sensitivity was detected in the CRT group after 1 month of OK. None of the midperipheral corneal changes produced in any of the groups
FIG. 4. Changes in corneal radius $(\mathrm{mm})$ produced by the 2 OK lenses along the horizontal and vertical meridians every $1 \mathrm{~mm}$ up to $4 \mathrm{~mm}$ from the center on the temporal or nasal side or in a superior or inferior direction.

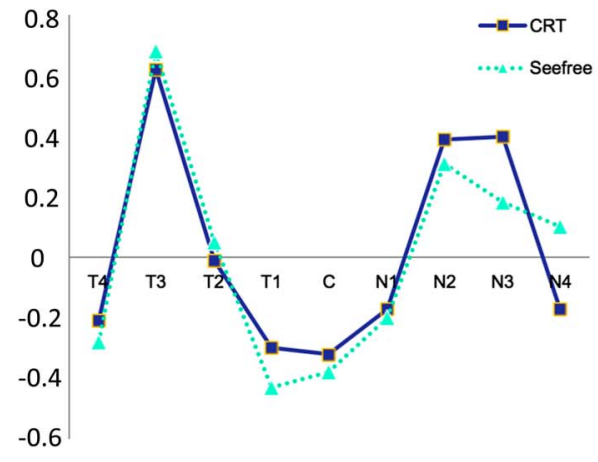

Horizontal meridian

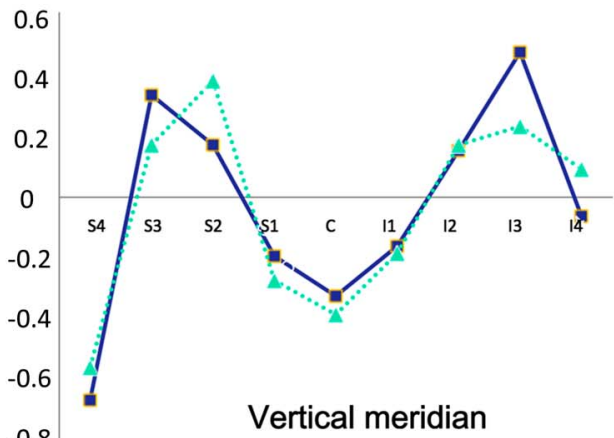

Vertical meridian 
was significant. This finding is in agreement with the findings of Lum et al., ${ }^{24,42}$ who postulated that changes in corneal sensitivity would be limited to the central cornea and midperipheral sensitivity would not be affected. Further, it has been proposed that the low sensitivity of the superior mid periphery is caused by neuronal adaptation to continuous pressure from the upper eyelid. ${ }^{43}$ During OK treatment, pressure is exerted over the entire central cornea such that the reduction detected in corneal sensitivity might be related to neuronal adaptation. Interestingly, no sensitivity effects of the SF lenses on the central cornea were produced after 1 month of OK though this could be the consequence of the smaller size of our SF group.

Although the Cochet-Bonnet esthesiometer is the standard clinical test for corneal sensitivity, ${ }^{41}$ a positive response is more dependent on the subject's attitude and apprehension. In effect, several precautions must be taken to ensure the reliability of measurements such as occasional feint approaches with the monofilament. Our corneal sensitivity results would have been enhanced by the use of a non-contact pneumatic esthesiometer, which has proved to be a more conclusive instrument. ${ }^{44}$ However, this instrument was not available at our laboratory.

After 1 month of OK treatment, nerve density and corneal sensitivity were unrelated both for the central and midperipheral cornea in all the subject groups. In line with this finding, Patel et al., ${ }^{19}$ who measured corneal sensitivity using a Cochet-Bonnet esthesiometer and examined SBNP nerve fiber bundles in healthy corneas and corneas of contact lens wearers, suggested that the drop in corneal sensitivity observed in contact lens wearers could reflect a functional change in nerves rather than a change in nerve density. In contrast, Rosenberg et al. ${ }^{21}$ reported both reduced corneal sensitivity and SBNP fibers in patients with type 1 diabetes, though this positive correlation was weak. Subsequently, Darwish et $\mathrm{al}^{20}$ also reported a reduction in nerve numbers and corneal sensitivity in patients who had undergone corneal surgery. However, although in these 2 studies, corneal sensitivity was resolved 3 months after the procedure, subbasal nerves counts were still abnormal 6 months later. However, given that patients differed in that they either had a systemic disease or had undergone irreversible corneal treatment, we propose that a functional change in corneal nerves is the main factor underlying the drop produced in corneal sensitivity. This determines a need to check the reversibility of nerve abnormalities after OK lens removal.

The SBNP has been fully described in normal human corneas ${ }^{28,34}$ and in patients undergoing OK treatment. ${ }^{14}$ In this study, we examined this living nerve structure in normal corneas and in the corneas of individuals who had worn OK lenses for a shortterm period using objective procedures to assess several variables. Although the size of the corneal area observed by IVCM was smaller than that examined by others, ${ }^{14,28}$ the large sample considered here provides evidence that overnight $\mathrm{OK}$ lens wear alters some parameters observed in healthy eyes. Moreover, our findings indicate that these changes may vary for different OK lens designs. Monitoring such changes in our treatment groups in the longer term would serve to determine whether these effects worsen, normalize, or remain steady throughout OK treatment. The low statistical power of our comparisons suggests that central sensitivity, central nerve length, and peripheral SBNP thickness values must be interpreted with caution. In conclusion, our study offers useful data regarding the anatomical effects of a short period of $\mathrm{OK}$ on the corneal SBNP and provides direction for future studies designed to assess the longer-term effects of this noninvasive refractive therapy procedure.

\section{ACKNOWLEDGMENTS}

The authors thank Óscar Gómez-Calderón, Ph.D. for resolving issues related to Matlab, Ignacio Rada for critical reading of the manuscript and the laboratories Interlenco (Madrid, Spain), and Conóptica (Barcelona, Spain) for their technical support.

\section{REFERENCES}

1. Sorbara L, Fonn D, Simpson T, et al. Reduction of myopia from corneal refractive therapy. Optom Vis Sci 2005;82:512-518.

2. Berntsen DABJ, Mitchell GL. The effect of overnight contact lens corneal reshaping on higher-order aberrations and best-corrected visual acuity. $O p$ tom Vis Sci 2005;82:490-497.

3. Felipe-Marquez G, Nombela-Palomo M, Cacho I, et al. Accommodative changes produced in response to overnight orthokeratology. Graefes Arch Clin Exp Ophthalmol 2015;253:619-626.

4. Villa-Collar C, Gonzalez-Meijome JM, Queiros A, et al. Short-term corneal response to corneal refractive therapy for different refractive Targets. Cornea 2009;28:311-316.

5. Barr JT, Pall B, Szczotka LB, et al. Corneal endothelial morphology results in the Menicon Z 30-day continuous-wear contact lens clinical trial. Eye Contact Lens 2003;29:14-16.

6. Stillitano I, Schor P, Lipener C, et al. Long-term follow-up of orthokeratology corneal reshaping using wavefront aberrometry and contrast sensitivity. Eye Contact Lens 2008;34:140-145.

7. Stillitano IG, Chalita MR, Schor P, et al. Corneal changes and wavefront analysis after orthokeratology fitting test. Am J Ophthalmol 2007;144:378-386.

8. Gonzalez-Meijome JM, Villa-Collar C, Queiros A, et al. Pilot study on the influence of corneal biomechanical properties over the short term in response to corneal refractive therapy for myopia. Cornea 2008;27:421-426.

9. Chen D, Lam AKC, Cho P. A pilot study on the corneal biomechanical changes in short-term orthokeratology. Ophthalmic Physiol Opt 2009;29: 464-471

10. Nichols JJ, Marsich MM, Nguyen M, et al. Overnight orthokeratology. Optom Vis Sci 2000;77:252-259.

11. Nieto-Bona A, Gonzalez-Mesa A, Nieto-Bona MP, et al. Short-term effects of overnight orthokeratology on corneal cell morphology and corneal thickness. Cornea 2011;30:646-654.

12. Lum E, Swarbrick H. Fibrillary lines in overnight orthokeratology. Clin Exp Optom 2007;90:299-302.

13. Nieto-Bona A, Gonzalez-Mesa A, Nieto-Bona MP, et al. Long-term changes in corneal morphology induced by overnight orthokeratology. Curr Eye Res 2011;36:895-904.

14. Lum E, Golebiowski B, Swarbrick HA. Mapping the corneal sub-basal nerve plexus in orthokeratology lens wear using in vivo laser scanning confocal microscopy. Invest Ophthalmol Vis Sci 2012;53:1803-1809.

15. Gonzalez-Perez J, Villa-Collar C, Sobrino Moreiras T, et al. Tear film inflammatory mediators during continuous wear of contact lenses and corneal refractive therapy. Br J Ophthalmol 2012;96:1092-1098.

16. Hazlett LD, McClellan SM, Hume EBH, et al. Extended wear contact lens usage induces Langerhans cell migration into cornea. Exp Eye Res 1999;69: 575-577.

17. Zhivov A, Stave J, Vollmar B, et al. In vivo confocal microscopic evaluation of Langerhans cell density and distribution in the corneal epithelium of healthy volunteers and contact lens wearers. Cornea 2007;26:47-54.

18. Hiraoka T, Kaji Y, Okamoto F, et al. Corneal sensation after overnight orthokeratology. Cornea 2009;28:891-895.

19. Patel SV, McLaren JW, Hodge DO, et al. Confocal microscopy in vivo in corneas of long-term contact lens wearers. Invest Ophthalmol Vis Sci 2002; 43:995-1003.

20. Darwish T, Brahma A, O'Donnell C, et al. Subbasal nerve fiber regeneration after LASIK and LASEK assessed by noncontact esthesiometry and in vivo confocal microscopy: Prospective study. J Cataract Refract Surg 2007;33:1515-1521.

21. Rosenberg ME, Tervo TMT, Immonen IJ, et al. Corneal structure and sensitivity in type 1 diabetes mellitus. Invest Ophthalmol Vis Sci 2000;41: 2915-2921. 
22. Tavakoli M, Boulton AJM, Efron N, et al. Increased Langerhans cell density and corneal nerve damage in diabetic patients: Role of immune mechanisms in human diabetic neuropathy. Cont Lens Anterior Eye 2011;34:7-11.

23. Patel DV, Tavakoli M, Craig JP, et al. Corneal sensitivity and slit scanning in vivo confocal microscopy of the subbasal nerve plexus of the normal central and peripheral human cornea. Cornea 2009;28:735-740.

24. Lum E, Golebiowski B, Swarbrick H. The recovery of corneal sensitivity and nerve morphology changes in orthokeratology. Invest Ophthalmol Vis Sci 2014;55:E-Abstract 4660.

25. Petropoulos IN, Manzoor T, Morgan P, et al. Repeatability of in vivo corneal confocal microscopy to quantify corneal nerve morphology. Cornea 2013;32:E83-E89.

26. Lawrenson JG, Ruskell GL. Investigation of limbal touch sensitivity using a Cochet-Bonnet aesthesiometer. Br J Ophthalmol 1993;77:339-343.

27. Stachs O, Zhivov A, Kraak R, et al. In vivo three-dimensional confocal laser scanning microscopy of the epithelial nerve structure in the human cornea. Graefes Arch Clin Exp Ophthalmol 2007;245:569-575.

28. Patel DV, McGhee CNJ. Mapping of the normal human corneal sub-basal nerve plexus by in vivo laser scanning confocal microscopy. Invest Ophthalmol Vis Sci 2005;46:4485-4488.

29. Misra S, Craig JP, McGhee CNJ, et al. Interocular comparison by in vivo confocal microscopy of the 2-dimensional architecture of the normal human corneal subbasal nerve plexus. Cornea 2012;31:1376-1380.

30. Abramoff MD, Magalhaes PJ, Ram SJ. Image processing with ImageJ. Biophoton Int 2004;11:36-42.

31. Meijering E. Neuron tracing in perspective. Cytometry A 2010;77A:693-704.

32. Cottrell P, Ahmed S, James C, et al. Neuron $J$ is a rapid and reliable open source tool for evaluating corneal nerve density in herpes simplex keratitis. Invest Ophthalmol Vis Sci 2014;55:7312-7320.

33. Kallinikos P, Berbanu M, O'Donnell C, et al. Corneal nerve tortuosity in diabetic patients with neuropathy. Invest Ophthalmol Vis Sci 2004;45:418-422.
34. Oliveira-Soto L, Efron N. Morphology of corneal nerves in soft contact lens wear. A comparative study using confocal microscopy. Ophthalmic Physiol Opt 2003;23:163-174.

35. He J, Bazan NG, Bazan HEP. Mapping the entire human corneal nerve architecture. Exp Eye Res 2010;91:513-523.

36. Hamrah P, Zhang Q, Liu Y, et al. Novel characterization of MHC class IInegative population of resident corneal Langerhans cell-type dendritic cells. Invest Ophthalmol Vis Sci 2002;43:639-646.

37. Tuisku IS, Konttinen YT, Konttinen LM, et al. Alterations in corneal sensitivity and nerve morphology in patients with primary Sjogren's syndrome. Exp Eye Res 2008;86:879-885.

38. Sindt CW, Grout TK, Critser DB, et al. Dendritic immune cell densities in the central cornea associated with soft contact lens types and lens care solution types: A pilot study. Clin Ophthalmol 2012;6:511-519.

39. Auran JD, Koester CJ, Kleiman NJ, et al. Scanning slit confocal microscopic observation of cell morphology and movement within the normal human anterior cornea. Ophthalmology 1995;102:33-41.

40. Torii H, Yan ZM, Hosoi J, et al. Expression of neurotrophic factors and neuropeptide receptors by Langerhans cells and the Langerhans cell-like cell line XS52: Further support for a functional relationship between Langerhans cells and epidermal nerves. J Invest Dermatol 1997;109: 586-591.

41. Booranapong $\mathrm{W}$, Tanthuvanit $\mathrm{P}$, Suwannik A. Corneal sensitivity in normal Thai population. Siriraj Med J 2005;57:262-265.

42. Lum E, Golebiowski B, Gunn R, et al. Corneal sensitivity with contact lenses of different mechanical properties. Optom Vis Sci 2013;90:954-960.

43. Draeger J. Topography of corneal sensitivity. In: Draeger J, ed. Corneal Sensitivity: Measurement and Clinical Importance. New York, NY, Springer-Verlag Wien, 1984, pp 40-41.

44. Murphy PJ, Patel S, Marshall J. A new non-contact corneal aesthesiometer (NCCA). Ophthalmic Physiol Opt 1996;16:101-107. 